\title{
Observations and Monte Carlo simulation of the Princess Sirindhorn Neutron Monitor at a vertical rigidity cutoff of $16.8 \mathrm{GV}$
}

\section{Pierre-Simon Mangeard*}

National Astronomical Research Institute of Thailand (NARIT), Chiang Mai 50200, Thailand. Department of Physics, Faculty of Science, Mahidol University, Bangkok 10400, Thailand.

E-mail: psmangeardegmail.com

\section{David Ruffolo, Alejandro Sáiz, Suttiwat Madlee}

Department of Physics, Faculty of Science, Mahidol University, Bangkok 10400, Thailand.

E-mail:david.ruf@mahidol.ac.th, alejandro.sai@mahidol.ac.th,

suttiwat.mad@mahidol.ac.th

\section{Tanin Nutaro}

Department of Physics, Faculty of Science, Ubon Ratchathani University, Ubon Ratchathani 34190, Thailand.

E-mail: tnutaro@yahoo.com

Neutron monitors (NMs) are large ground-based instruments for precise time tracking of the variations in the Galactic cosmic ray (GCR) flux at the GeV-range. NMs count the secondary particles (mostly neutrons) issued from the interaction of the cosmic rays in the Earth's atmosphere. The sensitivity to GCR variations depends on the geomagnetic cutoff at the location of measurement as well as on the altitude of detection. Since late 2007, the Princess Sirindhorn Neutron Monitor (PSNM), at the summit of Doi Inthanon, Thailand's highest mountain (2565 m altitude), has recorded the flux of galactic cosmic rays with the world's highest vertical rigidity cutoff for a fixed station, $16.8 \mathrm{GV}$. We present here the observations of PSNM since the beginning of its operation. We have also developed Monte Carlo simulations of cosmic ray interactions in the atmosphere and in PSNM (with its surroundings), which includes the tracing of cosmic ray trajectories through Earth's magnetic field to model the cosmic ray suppression at low rigidity at the location of PSNM. The simulated count rate is in reasonable agreement with the data. Variations of the GCR flux, such as the solar modulation effect, are investigated for the first time with a fixed ground-based NM at a such high geomagnetic cutoff, and the observed solar modulation is much weaker than predicted by the force field model with $\Phi$ inferred from NM data at low cutoff.

The 34th International Cosmic Ray Conference,

30 July- 6 August, 2015

The Hague, The Netherlands

\footnotetext{
* Speaker.
} 


\section{Introduction}

Since the middle of the $20^{\text {th }}$ century, neutron monitors (NM) have made unique and precise measurements of variations of the Galactic cosmic ray (GCR) flux. Many of the NMs are located at high latitude with a low geomagnetic cutoff rigidity, near or below the atmospheric cutoff of $1 \mathrm{GV}$ for a detector near the poles. Those locations allow them to be sensitive to variations of the GCR flux as well as to relativistic solar energetic particles that can be produced during solar storms. The Princess Sirindhorn Neutron Monitor (PSNM) is a relatively new ground based neutron detector in operation since December, 2007. Located at the top of Doi Inthanon, the highest mountain in Thailand $\left(18.59^{\circ} \mathrm{N}, 98.49^{\circ} \mathrm{E}, 2565 \mathrm{~m}\right)$ and at a vertical rigidity cutoff of $16.8 \mathrm{GV}$, PSNM has the objective to extend the range of sensitivity of the worldwide neutron monitor network. The period of operation of PSNM covers the last solar minimum to the present solar maximum and its data give the opportunity for the first time to study the solar modulation at a such high rigidity cutoff with a fixed station. The key component to analyze data from a NM is the so-called "yield function", which describes the detector response to primary particles at the top of the Earth's atmosphere. The yield function depends on the size of the detector, its characteristics, its location and its altitude. Great efforts have been made towards parameterizing this response. The simulated yield functions $[8,12]$ agree fairly well at high rigidity $(>30 \mathrm{GV})$. However, under $10 \mathrm{GV}$ they can still differ up to $\sim 100 \%$ due to differences in the chosen hadronic generator [13], detector properties and/or methodology. The empirical determination [6] using latitude survey and spacecraft data gives a more precise measurement at low rigidity but the energy range is limited by the maximum geomagnetic cutoff at sea level, $\sim 17 \mathrm{GV}$. Using the FLUKA simulation package [5, 9], we developed Monte Carlo (MC) simulations of the interaction of the primary cosmic rays in a realistic atmosphere at the location and the altitude of PSNM and determined the yield function of the detector including the surroundings of the station. We summarize in section 2 the operational status of PSNM and its observations from December, 2007 to the end of 2014. The computed yield function and the simulated solar induced variations are presented in section 3 .

\section{Observations}

The PSNM is a "18NM64" detector at Doi Inthanon with 18 B-10 proportional counter tubes in the standard NM64 configuration [7]. The tubes are equipped with electronics developed by the Bartol Research Institute, University of Delaware, USA. The values of the electronic dead time, $t_{d}$, for each tube range from 18 to $29 \mu \mathrm{s}$. The data used in the present work were recorded from December 9, 2007 to December 31, 2014, and the PSNM has continued to operate since then. The typical hourly count rate of $2.2 \times 10^{6}$ corresponds to a count rate of $\sim 34 \mathrm{~Hz}$ per tube. The hourly count rates were recorded with a very good duty cycle of $99.0 \%$ over more than 7 years (Table 1). The main causes of missing hours are the periods of maintenance, upgrades and power outages.

It is well known that a neutron monitor count rate depends strongly on the barometric pressure. The common correction depends on two parameters: $C_{p}=C e^{\beta\left(p-p_{\text {ref }}\right)}$, where $p$ is the pressure, $C$ the uncorrected count rate, $C_{p}$ the corrected count rate, $p_{\text {ref }}$ the reference pressure and $\beta$ the pressure correction coefficient. The values of $\left(p_{\text {ref }}, \beta\right)$ depend on the location, the altitude and the characteristics of the detector. At Doi Inthanon, we empirically determined $\beta=0.854 \% \mathrm{mmHg}^{-1}$ 


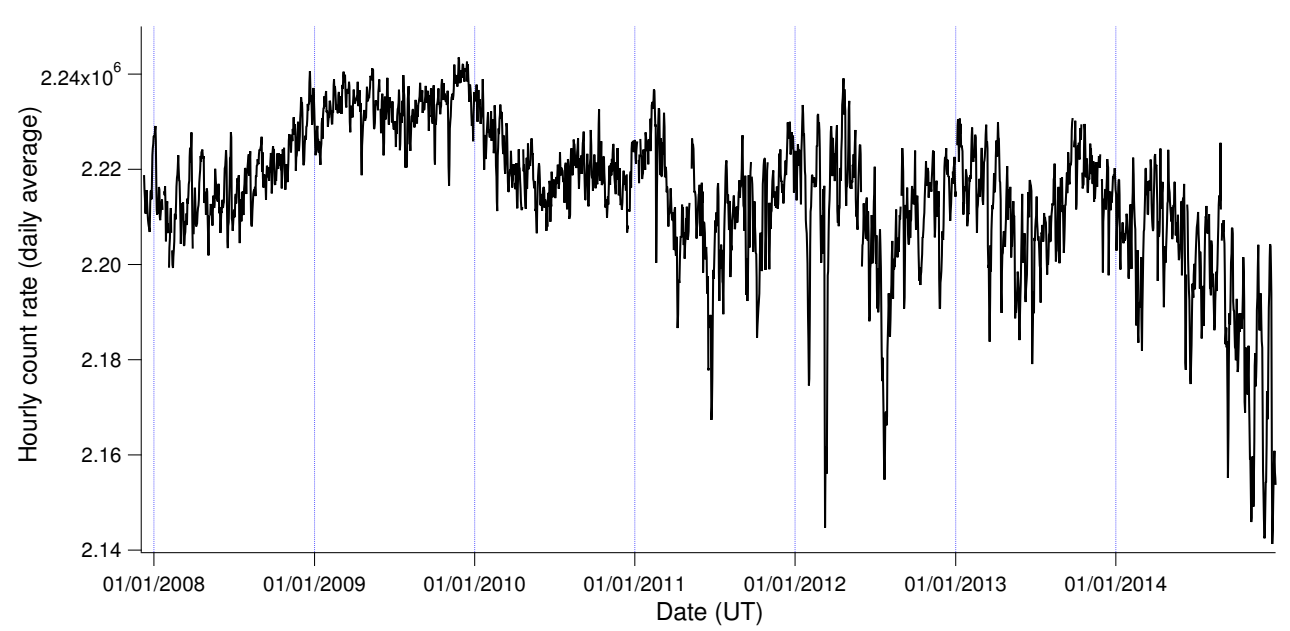

Figure 1: Hourly count rate of the PSNM at Doi Inthanon, Thailand from December 9, 2007 to December 31,2014 . The hourly count rates were corrected for pressure and then were combined into daily averages.

\begin{tabular}{|l|ccccccccc|}
\hline Year & $2007 *$ & 2008 & 2009 & 2010 & 2011 & 2012 & 2013 & 2014 & All \\
\hline Duty cycle (\%) & 100 & 98.7 & 99.4 & 99.2 & 97.8 & 99.4 & 98.8 & 99.4 & 99.0 \\
\hline
\end{tabular}

Table 1: Yearly duty cycle of PSNM since the start of operation. *Data for the period December 9-31, 2007.

and use $p_{\text {ref }}=563 \mathrm{mmHg}$. The corrected count rate from December 9, 2007 is shown in Figure 1. A long term variation is observed with the highest (lowest) average count rate of $2.2435 \times 10^{6}$ $\left(2.1413 \times 10^{6}\right)$ on November 25,2009 (December 23, 2014). This matches the general variations of the solar activity over the period of interest. The short term variations were limited $(\lesssim 1 \%)$ during the long period of low solar activity in 2008-2009. Larger variations are visible during the present solar maximum. Forbush decreases were observed with the largest decrease of $\sim 3.5 \%$ in March, 2012.

\section{Monte Carlo simulation}

\subsection{Method}

We used the FLUKA (2011) Monte Carlo simulation software [5, 9] with the interaction model DPMJET-2.5 as recommended in the FLUKA manual (via the HEAVYNUC option). The PHOTONUC option (for photonuclear processes) was activated. The method is composed of three stages: $i$ ) the air shower development induced by the primary cosmic rays is simulated and the secondary particle (SP) fluxes are extracted at the altitude of the detector plus six meters, ii) with a second simulation, the sensitivity of the 18NM64 within the station is determined for the main types of SPs and iii) the outputs of both simulations are combined to calculate the response of PSNM. The atmospheric profiles of the present work combine information from two models: the Global Data Assimilation System (GDAS, http://ready.arl.noaa.gov/gdas1.php) at low altitude (which includes moist air), and the Naval Research Laboratory Mass Spectrometer, Incoherent Scatter Radar 

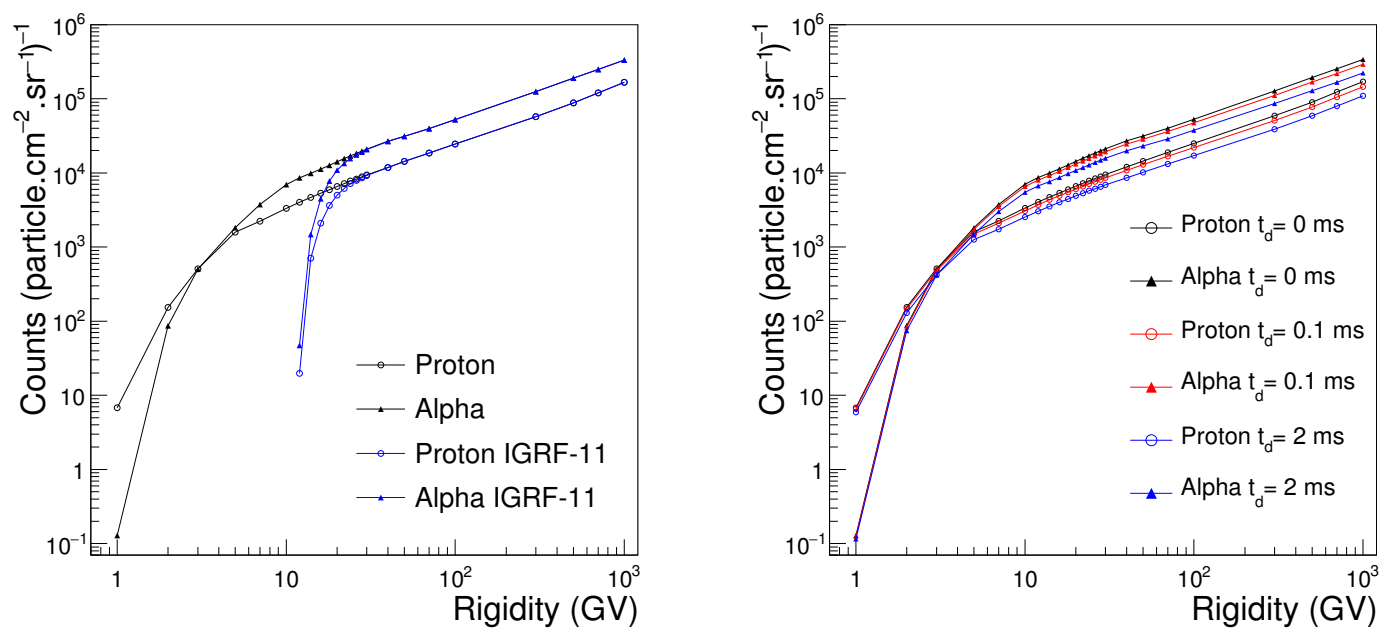

Figure 2: Yield functions of the PSNM from MC simulation. (Left) Effect of the geomagnetic cutoff according to the IGRF-11 geomagnetic field model. The dead times used in the simulation are set separately for each tube according to measured values. (Right): Effect of the electronic dead time $t_{d}$. Here a single dead time is assumed for the 18 tubes.

Extended model (NRLMSISE-00 [15]) for dry air at higher altitude up to $72.5 \mathrm{~km}$. The total pressure was $563.4 \mathrm{mmHg}$ with a contribution of $4.7 \mathrm{mmHg}$ from the water vapor (atmospheric depth of $769.9 \mathrm{~g} \mathrm{~cm}^{-2}$ ). This corresponds to a typical atmosphere over Doi Inthanon during January (dry season). The fluxes of SPs were calculated for mono-energetic, isotropic fluxes of protons and alphas at the top of the 3D spherical atmosphere. The response of the 18NM64 to the SPs was estimated with a second simulation. A detailed geometry of the station was included inside the FLUKA geometry editor. Multiple sets of simulations were performed depending on the type of $\mathrm{SP}$ (proton, neutron, $\mu^{+}, \mu^{-}, \pi^{+}, \pi^{-}, e^{+}, e^{-}$and $\gamma$ ), its kinetic energy (from $1 \mathrm{meV}$ to $100 \mathrm{GeV}$ for neutrons) and its incidence direction among five zenith angles $\theta\left(0^{\circ}, 15^{\circ}, 30^{\circ}, 50^{\circ}\right.$ and $\left.70^{\circ}\right)$ and eight azimuthal angles $\varphi$ (from $0^{\circ}$ to $315^{\circ}$ ).

\subsection{Yield function}

In addition to the location and the type of the detector, several choices of parameters and methods used in the simulation can affect the computed yield functions, such as the geomagnetic field and the electronic dead time. The effects from the East-West asymmetry induced by the geomagnetic field were estimated. A direct tracing of each individual simulated primary particle through the geomagnetic field was performed using version 11 of the coefficients of the International Geomagnetic Reference Field (IGRF-11), actually by tracing its antiparticle backwards from the top of the atmosphere. Figure 2 (left) presents the yield functions (for protons and alphas) without a geomagnetic field and with the IGRF-11 applied. Despite a clear decrease, we can observe that the response at $12-17 \mathrm{GV}$ is not negligible. The yield functions are also significantly affected by the geomagnetic field up to $30 \mathrm{GV}(\sim 1.5 \%)$ and even at $40 \mathrm{GV}(\sim 0.1 \%)$. Due to this strong East-West asymmetry, the vertical cutoff value of $16.8 \mathrm{GV}$ must be used with care. 


\begin{tabular}{|c|l|c|cccccc|}
\hline \multirow{2}{*}{ Month/ $\Phi$} & \multirow{2}{*}{ Data } & \multicolumn{7}{|c|}{ Simulation input spectrum } \\
\cline { 4 - 9 } & & & PAMELA & US11 & WH09 & GM75 & WH03 & LA03 \\
\hline Dec. 2009/ & Count rate & $\mathbf{2 . 2 3 7}$ & 2.39 & 2.35 & 2.24 & 2.42 & 2.44 & 2.36 \\
255 MV & MC/obs. & - & 1.068 & 1.051 & 1.001 & 1.082 & 1.091 & 1.0545 \\
\hline \multirow{2}{*}{ Nov. 2014/ } & Count rate & $\mathbf{2 . 1 7 5}$ & - & 2.23 & 2.12 & 2.29 & 2.31 & 2.22 \\
646 MV & MC/obs. & - & - & 1.025 & 0.975 & 1.053 & 1.062 & 1.021 \\
& Decrease (\%) & $\mathbf{2 . 7 7}$ & - & 5.1 & 5.4 & 5.4 & 5.3 & 5.9 \\
\hline
\end{tabular}

Table 2: Comparison of the observed count rate $\left(\times 10^{-6} \mathrm{~h}^{-1}\right.$, monthly average $)$ with $\mathrm{MC}$ simulation results for six different primary spectra: the observed PAMELA proton spectrum for Dec. 2009 (the PAMELA alpha spectrum was measured during the period 2006-2008) [1,2] and the force-field model of solar modulation for the five LIS models from [11] and the $\Phi$ value from [16]. The decrease is from December, 2009 to November, 2014.

The electronic dead time, $t_{d}$, of a NM is one of the main parameters needed to achieve a precise simulation of the yield function or to intercalibrate multiple NMs [3], as $t_{d}$ is directly related to the multiplicity of the detector response [4]. For instance, a short dead time of few microseconds will allow the proportional counters to record the series of multiple counts produced by a single SP, whereas for a value of few milliseconds, the NM will be sensitive essentially to the first count only. We determined the yield function of the PSNM for 3 values of $t_{d}(0,0.1$, and $2 \mathrm{~ms})$, taken to be identical for the 18 tubes (right of Figure 2). At $20 \mathrm{GV}$, a decrease of $\sim 8-9 \%(\sim 25 \%$ ) can be observed for $t_{d}=0.1 \mathrm{~ms}\left(t_{d}=2 \mathrm{~ms}\right)$ relative to the perfect case of no dead time. At $1000 \mathrm{GV}$, the decrease is as high as $\sim 15 \%(\sim 35 \%)$.

\subsection{Solar modulation}

In this part, the primary particles were simulated uniformly for several ranges of kinetic energy per nucleon, separately for protons and for alphas. The same back-tracking of antiparticles through the geomagnetic field was used. The appropriate weights regarding the chosen GCR spectrum were applied post-simulation. The force-field approximation [10] is a simple model of solar modulation of the local interstellar spectrum (LIS). It allows us to parametrize the solar modulation with a single parameter $\Phi$ expressed in GV. At the top of the Earth's atmosphere, the flux can be expressed in particles $\mathrm{m}^{-2} \mathrm{~s}^{-1} \mathrm{sr}^{-1} \mathrm{GeV}^{-1}$ :

$$
j_{i, T}^{1 \mathrm{AU}}(P)=\left(\frac{P}{P+\Phi}\right)^{2} \times j_{i, T}^{\mathrm{LIS}}(P+\Phi)
$$

where $i$ is the type of particle, $P$ its rigidity, $T$ its kinetic energy per nucleon and $j_{i, T}^{\mathrm{LIS}}(P)$ the associated LIS. Note that $\Phi$ is changed according to the LIS model, following [11]. In the rest of this work, the value we report for the modulation parameter $\Phi=\Phi_{\mathrm{US} 11}$ corresponds to the LIS from [16]. In addition to those semi-theoretical models, we also used the proton spectra measured by the PAMELA spacecraft at specific epochs $[1,2]$ and a single spectrum they measured for alpha particles during 2006-2008 [2].

We compared the absolute simulated count rates with the observation for the period with the lowest solar modulation in December 2009 to reduce the uncertainty from the force field approxi- 


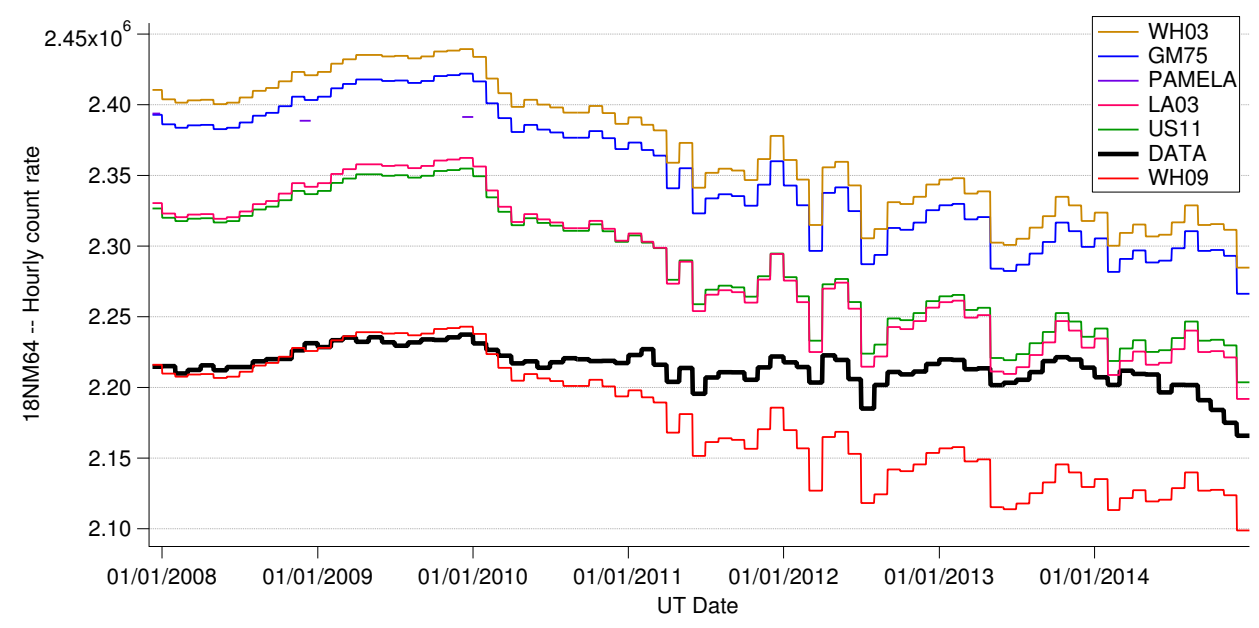

Figure 3: Variation of the hourly count rate (averaged monthly) of the PSNM at Doi Inthanon from December 2007 to December 2014 compared with the MC simulation results, based on input spectra from PAMELA or from the force field model of solar modulation for five different LIS models from [11]. The monthly values of the modulation parameter $\Phi$, as inferred from NM data at or below $6.3 \mathrm{GV}$ cutoff rigidity, were taken from http://cosmicrays.oulu.fi/phi/phi.html [16]. The simulated count rates based on the PAMELA data are shown for only 3 months. The observed solar modulation at Doi Inthanon is much weaker than expected from the force-field model with this $\Phi(t)$.

mation. The spectra from PAMELA were also available during that month. The results are shown in Table 2. The simulated Doi Inthanon count rate using PAMELA spectra overestimates the observed Doi Inthanon count rate of $\sim 6.8 \%$. Taking into account the $5-10 \%$ of uncertainty of the input PAMELA spectra measurement and the approximate determination of the input heavy nuclei contribution (simulated as $54.8 \%$ of the alpha spectrum), this gives us confidence in our MC simulation techniques. As mentioned before, the primary particles with $P<17 \mathrm{GV}$ contribute significantly to the count rate and represent $\sim 7 \%$ of the total count rate for the 4 sets of PAMELA spectra (from 2006 to 2009). We determined that about $60 \%$ of the counts are induced by primary protons at the top of the atmosphere. For this period of low solar activity, we estimated the median rigidity at Doi Inthanon as $\sim 35 \mathrm{GV}$.

More generally, the simulated count rates using the different LIS models all overestimate the observed count rate by $0.1 \%$ to $9.1 \%$. This level of variation between the models is expected [11]. Note here that about half of the difference between the models comes from the spectrum of the primary particles with $P>100 \mathrm{GV}$. The uncertainty of $26 \mathrm{MV}$ on the modulation parameter [16] implies an uncertainty lower than $0.5 \%$ in the simulated count rate. To study the effect of the solar modulation in the count rate at Doi Inthanon since 2007, we used the monthly modulation parameter calculated by the method from [16], based on NMs at cutoff $\leq 6.3 \mathrm{GV}$. Figure 3 presents the simulated monthly Doi Inthanon count rates for the force-field model for the five LIS models, compared with the actual PSNM observations. The simulated count rate determined using PAMELA spectra is also shown for the three available months. We see a good qualitative agreement between the observed and the simulated variations (short and long term). It is important to mention here that $\Phi$ is calculated using the variations of multiple NM count rates at different geomagnetic rigidity 
cutoffs, but all of them are lower than $6.3 \mathrm{GV}$. Within the force field approximation, the method can reproduce the variations of the GCR spectrum within 5-10\% [17] and a decrease of the GCR flux at low rigidity implies a decrease at higher rigidity. The PSNM observations confirm this. However, the decrease of the PSNM count rate from December, 2009 to November, 2014 is about half of the predicted decrease, as shown in the last line of Table 2 . The observed decrease is $\sim 2.8 \%$ while the simulations show a decrease of 5-6\% for the different LIS models tested in this work. The characteristics of the PSNM (high altitude, 18 tubes, high rigidity cutoff) give a statistically precise measurement of the count rate with a low uncertainty of $\sim 0.1 \%$. Thus, the observed variation at Doi Inthanon provides a direct measurement of the intensity of the solar modulation at $P \geq 20 \mathrm{GV}$. Despite a fairly good agreement at low rigidity, the force-field model with a modulation parameter extracted from the worldwide neutron monitor network at low rigidity cannot represent with sufficient accuracy the GCR spectrum at the top of the atmosphere at high rigidity.

The force-field model is spherically symmetric, and has a known deficiency of not including drifts and other effects related to solar magnetic polarity [14]. However recent latitude survey results indicate that relative to a polar monitor, the NM count rate at high cutoff rigidity varied more rapidly during the $A<0$ polarity preceding the recent solar maximum than expected from the force-field model [14]. Therefore, including solar magnetic polarity effects would worsen the disagreement between the model results and the PSNM observations at Doi Inthanon.

\section{Conclusions}

The Princess Sirindhorn Neutron Monitor operated since 2007 at the world's highest rigidity cutoff for a fixed station. Its observations cover the period of the last solar minimum and the current solar maximum and provide a good opportunity to study the effect of solar activity on the GCR flux at high rigidity $(P \sim 20 \mathrm{GV})$. We developed a Monte Carlo simulation of the interaction of the cosmic rays in a realistic atmosphere at the location of Doi Inthanon and determined the sensitivity of PSNM to the secondary particles. We showed that the effect of the electronic dead time on the yield function is larger at high rigidity, as expected, and also that it can induce large systematic errors in the yield function if not properly taken into account. We compared the observations with simulated count rates for multiple choices of the input cosmic ray spectrum at the top of the atmosphere. Agreement within 5-10 \% is observed between the data and all Monte Carlo results. This accuracy is comparable with other typical computed yield functions [6, 8, 12]. Finally, using the modulation parameter extracted from the worldwide neutron monitor network at low rigidity $(\leq 6.3 \mathrm{GV})$, we generated input spectra with the force-field model and derived expected variations of the PSNM monthly count rate between 5 and 6\% since 2007. The actual NM observations at Doi Inthanon show a much lower count rate variation of $\sim 2.8 \%$. This indicates that the forcefield approximation with $\Phi$ inferred from NM at low rigidity significantly overestimates the solar modulation of the GCR spectrum at $P \sim 20 \mathrm{GV}$.

\section{Acknowledgments}

We thank the Royal Thai Air Force and Chatchai Injai for their kind assistance with the observations, and Andrew Snodin for setting up and maintaining the computing cluster on which 
our simulations were performed. P.-S. M. was partially supported under the postdoctoral research sponsorship of Mahidol University.

\section{References}

[1] O. Adriani et al., PAMELA Measurements of Cosmic-Ray Proton and Helium Spectra, Science, 332, 69, doi: 10.1126/science.1199172 (2011).

[2] O. Adriani et al., Time Dependence of the proton flux measured by PAMELA during the 2006 July-2009 December Solar Minimum, Astrophys. J., 765:91, doi:10.1088/0004-637X/765/2/91 (2013).

[3] N. Aiemsa-ad et al., Measurement and simulation of neutron monitor count rate dependence on surrounding structure, J. Geophys. Res. Space Physics, 120, doi: 10.1002/2015JA021249 (2015).

[4] J. W. Bieber, J. M. Clem, M. L. Duldig, P. A. Evenson, J. E. Humble, and R. Pyle, (2004), Latitude survey observations of neutron monitor multiplicity, J. Geophys. Res., 109, A12106, doi:10.1029/2004JA010493 (2004).

[5] T. T. Bohlen, F. Cerutti, M. P. W. Chin, A. Fassò, A. Ferrari, P. G. Ortega, A. Mairani, P. R. Sala, G. Smirnov, and V. Vlachoudis, The FLUKA Code: developments and challenges for high energy and medical applications, Nuclear Data Sheets, 120, 211-214 (2014).

[6] R. A. Caballero-Lopez and H. Moraal, Cosmic-ray yield and response functions in the atmosphere, J. Geophys. Res., 117, A12103, doi:10.1029/2012JA011794 (2012).

[7] H. Carmichael, IQSY Instruction Manual No. 7, London: IQSY Secreatariat, (1964).

[8] J. Clem and L. Dorman, Neutron monitor response functions, Space Sci. Rev., 93, 335 (2000).

[9] A. Ferrari, P. R. Sala, A. Fassò and J. Ranft, FLUKA: a multi-particle transport code, CERN-2005-10, INFN/TC_05/11, SLAC-R-773 (2005).

[10] L. J. Gleeson and W. I. Axford, 1968, Solar modulation of Galactic cosmic rays, Astrophys. J., 154, 1011, (1968).

[11] K. Herbst et al. , On the importance of the local interstellar spectrum for the solar modulation parameter, J. Geophys. Res., 115, D00I20, doi:10.1029/2009JD012557 (2010).

[12] A. L. Mishev, I. G. Usoskin and G. A. Kovaltsov, Neutron monitor yield function: New improved computations, J. Geophys. Res., 118, 2783, doi:10.1002/jgra.50325 (2013).

[13] A. L. Mishev and P. I. Y. Velinov, Influence of hadron and atmospheric models on computation of cosmic ray ionization in the atmosphere - Extension to heavy nuclei, J. Atm. Solar-Terr. Phys., 120, 111, doi:10.1016/j.jastp.2014.09.007 (2014).

[14] W. Nuntiyakul et al., Latitude survey investigation of Galactic cosmic ray solar modulation during 1994-2007, Astrophys. J., 795:11, 2014 November 1.

[15] J. M. Picone, A. E. Hedin, D. P. Drob, A. C. Aikin, NRLMSISE-00 empirical model of the atmosphere: Statistical comparisons and scientific issues, http://ccmc.gsfc.nasa.gov/modelweb/models/nrlmsise00.php, J. Geophys. Res., 107, 1468, doi:10.1029/2002JA009430 (2002).

[16] I. G. Usoskin, G. A. Bazilevskaya and G. A. Kovaltsov, Solar modulation parameter for cosmic rays since 1936 reconstructed from ground-based neutron monitors and ionization, J. Geophys. Res., 116, A02104, doi:10.1029/2010JA016105 (2011).

[17] I. G. Usoskin et al., Force-field parameterization of the galactic cosmic ray spectrum: Validation for Forbush decreases, Advances in Sp. Res., 55, 2940, (2015). 